\title{
ORCID y Funder Registry de Crossref como infraestructuras de información disponibles para el seguimiento del flujo de financiación en investigación: el talón de Aquiles de las entidades financiadoras
}

ORCID and Crossref's Funder Registry as the available information infrastructures to monitor the flow of research funding: the Achilles heel of funding bodies

\author{
Miriam TOMÁs LóPEZ \\ Fundación Séneca, Región de Murcia y Facultad de Comunicación y Documentación de la Universidad de Murcia, \\ Campus Universitario de Espinardo-30100 Murcia (España), miriam.tomas1@um.es
}

\begin{abstract}
Resumen
Se presentan las principales infraestructuras de información que en un contexto de ciencia abierta tienen a su disposición las entidades que gestionan fondos públicos en investigación y que son necesarias para ampliar la trazabilidad de la financiación que conceden y medir el impacto de la misma. De esta forma las entidades financiadoras podrán diseñar sus políticas científicas dirigidas a las necesidades reales de la sociedad y contribuir a la transparencia en su gestión y al rendimiento de cuentas. Asimismo, se muestran las funcionalidades que ofrecen ORCID y Crossref para conectar los resultados con las actuaciones financiadas que han hecho posible su aparición y se analiza si son suficientes para poder completar el mapeo de las ayudas concedidas.
\end{abstract}

Palabras clave: Entidades financiadoras. Resultados de investigación. ORCID. Crossref. Subvenciones. Identificadores persistentes. Fondos públicos.

\section{Introducción}

Las agencias financiadoras de ayudas públicas a la I+D+i son instrumentos cuya misión es la de promocionar la investigación científica y técnica en todas las áreas de conocimiento mediante la asignación eficiente de los recursos públicos, el fomento de la colaboración entre los agentes del sistema de I+D+i y el apoyo a la generación de conocimiento científico y técnico, económico y social. Además de financiar, mediante la evaluación y seguimiento de las acciones que impulsan, son el medio para conseguir la transformación del sistema de investigación y mejorar las estrategias de las instituciones académicas y los organismos de investigación para que sean más eficientes a nivel mundial.

\begin{abstract}
This study presents the primary information infrastructures used in an open science context to manage public funding for research. The information infrastructures are necessary to increase the traceability of awarded funding and to measure its impact, allowing funding agencies to design their scientific policies to be aimed at the real needs of society and to contribute to the transparency in their management and accountability. The communication also highlights the functionalities offered by ORCID and Crossref to connect results with financial actions made possible by the funding, and analyses whether they are sufficient to complete the mapping of the grants awarded.
\end{abstract}

Keywords: Funding agencies. Research funding. ORCID. Crossref. Grants. Persistent identifiers. Public funds.

Entre los retos principales de las entidades que gestionan fondos públicos en investigación está la evaluación del rendimiento de sus programas y actuaciones y la medición del retorno de la inversión realizada en los mismos. Ambos están encaminados a diseñar futuras políticas científicas dirigidas a las necesidades reales de la sociedad y a definir mandatos de acceso abierto para poner a disposición de la sociedad los resultados de las investigaciones que financian contribuyendo de esta forma a la transparencia en su gestión y al rendimiento de cuentas como receptora de dichos resultados. La ciencia abierta sin duda ha facilitado a las agencias financiadoras implicadas en este movimiento a la consecución de ambos objetivos proporcionándoles las herramientas necesarias para mejorar sus flujos de trabajo y procesos (De-Castro, 2017) y permitiendo que la 
inversión realizada por las agencias contribuya a la aceleración del conocimiento, la transferencia directa del mismo a la sociedad y una mayor visibilidad de la inversión pública en investigación.

En este entorno de ciencia abierta cada vez son más las agencias financiadoras interesadas en participar en ella intercambiando sus datos de financiación con el resto de agentes implicados por la necesidad que tienen de monitorizar los resultados de las investigaciones que apoya y hacer un seguimiento del cumplimiento por parte de los beneficiarios de las ayudas de sus políticas de gestión y acceso abierto. Hasta el momento las entidades pequeñas, salvo algunas excepciones, este seguimiento lo hacen manualmente a través de los informes de justificación científica que presentan los beneficiarios de las ayudas en el momento de finalizar el periodo de la subvención, lo que supone una tarea tediosa para el beneficiario y poco práctica para la entidad financiadora por la naturaleza de los informes cuyo formato impide el manejo y explotación de la información para su uso posterior en la evaluación de sus programas y toma de decisiones, así como la posibilidad de comparar los datos con los de otras agencias análogas para poder analizar las tendencias en políticas públicas en materia de investigación.

En el presente artículo se presentan los principales recursos e infraestructuras de información que en un contexto de ciencia abierta tienen a su disposición las entidades que gestionan fondos públicos en investigación y que necesitan usar y en algunos casos implementar en sus sistemas de flujo de trabajo para poder hacer un seguimiento de la financiación que conceden y medir el retorno de su inversión en términos de resultados publicados por los beneficiarios. Asimismo, se muestran sus funcionalidades dirigidas a las entidades financiadoras y si son suficientes para poder completar la trazabilidad de las ayudas concedidas.

\section{El papel de los identificadores persistentes (PIDs)}

Como indica De-Castro (2018) para evaluar el impacto de la investigación que financian las agencias es necesario conectar los resultados con las actuaciones financiadas que han hecho posible su aparición, argumento que es reforzado por una encuesta realizada por ORCID en 2019 en la que se concluía que uno de los principales desafíos a los que se enfrentan las entidades financiadoras es la vinculación de las subvenciones a las actividades y resultados de investigación posteriores (Meadows, Brown, Demeranville y Haak, 2019). Para el establecimiento de dicho vínculo los PIDs juegan un papel fundamental porque son códigos que identifican de forma única los elementos centrales en la infraestructura científica (investigadores, resultados de investigación, organizaciones y subvenciones) y, a su vez, actúan de conectores de información confiable al facilitar la interoperabilidad de los metadatos asociados a dichos elementos (Meadows y Haak, 2018), brindando a las entidades de financiación la posibilidad de mapear al detalle el flujo de la financiación, su búsqueda, identificación y análisis dentro del ecosistema de la investigación.

Los beneficios de incorporar en los sistemas de flujo de trabajo de la entidad financiadora los PIDs de organización, subvenciones e investigadores, todos ellos conectados a los metadatos en el DOI (Digital Object Identifier) de la publicación, hace que los procesos sean más rápidos, fáciles y confiables (Meadows, Haak y Brown, 2019). Al mismo tiempo, le permite al investigador agilizar la tramitación de su solicitud porque se autocompletan algunos de los campos requeridos en la misma con información normalizada así como la elaboración de informes de justificación científica más precisos y automatizados una vez finalizado el periodo de concesión. Estas conexiones abiertas, digitales y rastreables muestran qué están publicando los beneficiarios de las ayudas, en qué fuentes de difusión lo están haciendo y si comparten los datos y sus publicaciones en acceso abierto siguiendo los mandatos de la entidad, lo que conlleva un salto cualitativo en los procesos de gestión, evaluación, comunicación y medición de la investigación de la entidad financiadora.

Actualmente la comunidad científica y en particular las agencias financiadoras tienen a su disposición una amplia y sofisticada infraestructura abierta, global e interoperable de sistemas de identificación persistentes para algunos elementos clave que forman parte del flujo de datos en investigación. Están los identificadores persistentes para investigadores y colaboradores (ORCID iD), para datos y software (DataCite DOI), para los resultados de la investigación como artículos de revistas, preimpresiones, actas de conferencias, revisiones por pares, monografías y estándares (Crossref DOI), para financiadores (Open Funder Registry IDs) (Bilder, 2017) y para organizaciones de investigación (ROI IDs), entre otros. La información de investigación sólo es útil si está conectada, accesible y se puede compartir con la comunidad científica y eso supone que los sistemas de datos estén conectados entre sí a través de los mencionados PIDs (Meadows y Haak, 2018). Su uso y combinación potencian la fiabilidad de la información ofreciendo a las entidades financiadoras información confiable para evaluar sus programas de $I+D+i$ y definir futuras 
políticas científicas orientadas a las necesidades reales de la sociedad.

\section{Crossref}

\subsection{Funder Registry de Crossref}

Una de las herramientas que tienen a su disposición las entidades financiadoras para hacer el seguimiento de los resultados de las investigaciones que impulsa es Funder Registry que administra Crossref. Es un registro que proporciona una taxonomía única de financiadores que es incorporada junto con los metadatos de financiación asociados al sistema de seguimiento de los trabajos científicos de las revistas. Este registro se lanzó en 2012 (con el apoyo de Elsevier) y ha crecido hasta llegar a 20.000 financiadores en todo el mundo, está disponible bajo una licencia CC0 y su uso es gratuito (Mejias, 2020).

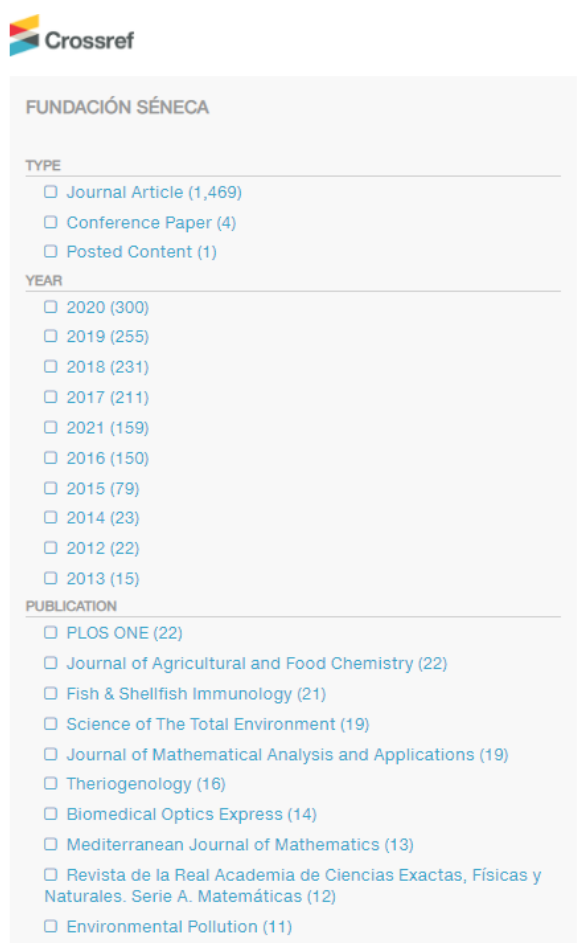

Figura 1. Fuentes utilizadas por los beneficiarios

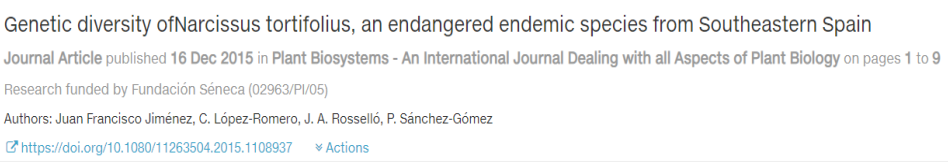

Figura 2. Registro con el código de subvención

Los beneficiarios de ayudas públicas están obligados a reconocer las fuentes de financiación y las contribuciones a su investigación en los resultados que publican. Para ello mencionan en alguna parte de su publicación (por lo general en la sección de agradecimientos) la agencia financiadora y el código de subvención que ha hecho posible dicha contribución. Los editores por su parte incluyen esta información estandarizada en sus metadatos cuando registran el contenido con Crossref conectándolos con el identificador digital único (ID) de la entidad financiadora que ofrece Funder Registry y enviándola posteriormente a Crossref quien la pone a disposición de todas las partes implicadas en el proceso (organizaciones, agencias financiadoras, autores y editores) para un seguimiento más eficiente, confiable y transparente de los resultados de la investigación (Crossref, n.d.).

Supongamos que una entidad financiadora regional como es la Fundación Séneca necesita saber las fuentes de publicación utilizadas por sus beneficiarios para comprobar el cumplimiento de sus mandatos de acceso abierto. Como dicha entidad ya está registrada en Funder Registry con un ID único, al empezar a introducir su nombre en la caja de búsqueda aparece la entidad para que pueda ser seleccionada, a continuación, se muestran todas aquellas publicaciones en las que en sus metadatos de financiación aparece la Fundación Séneca como patrocinador, y si el autor ha mencionado en su contribución el código de subvención de la ayuda también aparecerá en dichos metadatos (Figuras 1 y 2 ).

\subsection{EI ID de subvención de Crossref}

Una vez identificado el resultado de la investigación con la entidad financiadora habría que descender un nivel más hasta poder conectar dicho resultado con la actuación concreta de la que surge y su código de subvención, siendo éste un indicador clave para que las entidades financiadoras puedan monitorizar los resultados de forma confiable y hacer un uso posterior de los datos que arrojan estas conexiones.

Vincular los resultados de la investigación con sus códigos de subvención no es una tarea sencilla ya que a pesar de que las agencias en sus bases de convocatoria instan a sus beneficiarios a que las mencionen y cómo deben hacerlo, sigue dependiendo de la voluntad de los investigadores citar a sus patrocinadores. Cuando lo hacen como indican Kiley, Frentrop y Hendricks (2018) frecuentemente cometen errores u omiten la información del número de subvención y si lo mencionan como cada entidad financiadora utiliza su propio código de subvención, estos números no son únicos. Crossref resuelve este problema con un sistema en el que cada subvención concedida tiene un identificador global único que puede vincularse sin ambigüedades a todos los 
resultados (artículos, datos, código, materiales, patentes, etc.) que surjan de ella.

En 2019, el financiador Wellcome registró los primeros ID de subvención con Crossref. Un registro de subvenciones que permite a los financiadores y a la comunidad científica en general conectar las ayudas con los resultados y las actividades de investigación a través de los identificadores únicos (IDs) para subvenciones. Estos IDs conectan directamente los resultados de la investigación con los códigos de las subvenciones permitiendo completar la trazabilidad de las ayudas concedidas por una entidad financiadora una vez que ésta proporcione el ID de subvención y se pase a los metadatos de la publicación, de esta forma el financiador podrá buscar un ID de subvención y ver qué resultados se han asociado a la misma y a los editores les ayudará a generar reconocimientos de financiación más precisos (Lammey, 2020).

Actualmente es una iniciativa relativamente joven en la que Crossref sigue trabajando con un amplio grupo de financiadores de investigación, especialmente en la salida de metadatos de subvenciones a través de sus APIs (Interfaz de programación de aplicaciones) abiertas y herramientas de búsqueda; $y$ en el desarrollo de un widget para que las plataformas de envío de manuscritos puedan adaptar las subvenciones de manera coherente para investigadores, editores y otros (Crossref, 2021). Por el momento, las entidades financiadoras ya pueden registrar sus IDs de subvención con Crossref y en breve estos metadatos comenzarán a fluir a través de otros sistemas. Una vez incluidas por los editores en los metadatos de la publicación como hacen con los IDs de las entidades financiadoras los autores podrán seleccionar los datos específicos de la subvención cuando envían sus contribuciones a los editores.

\subsection{La membresía de Crossref} para organizaciones

Una organización para obtener un prefijo DOI (Identificado de Objetos Digitales) de Crossref y poder así crear enlaces únicos y persistentes para distribuir sus metadatos a otras organizaciones debe ser miembro de Crossref y pagar una cuota anual y otra única por cada elemento de contenido registrado.

En el caso de las entidades financiadoras para obtener los DOI de subvención sus cuotas anuales de membresía son más bajas que las del resto de miembros, pero el coste por registro de subvención es más alto. Las cuantías varían en función de la financiación anual de la subvención (Crossref, 2019).

\section{ORCID}

\subsection{Identificador ORCID para investigadores}

Para que las agencias financiadoras puedan hacer un seguimiento de los resultados de las investigaciones que financian es necesario que los solicitantes de sus convocatorias públicas dispongan de un identificador ORCID para que puedan tramitar la ayuda y hagan uso de él cuando publiquen artículos en revistas u otro tipo de resultado de investigación.

ORCID es una organización internacional sin ánimo de lucro que proporciona a los investigadores de forma gratuita un identificador digital único y persistente, conocido como ORCID iD, que los vincula con su trabajo y les permite compartir sus actividades con los sitios web participantes, incluidas las pasarelas científicas (Arnold, Dahan, Hart, y Soriano, 2019).

La información registrada en ORCID se estructura en distintas secciones: información personal, publicaciones científico-técnicas, filiación institucional, formación, fuentes de financiación, etc. cuyo contenido puede ser editado y modificado por el investigador usando su usuario y password. Concretamente en el apartado de fuentes de financiación el usuario puede registrar la información manualmente seleccionado las entidades financiadoras y las subvenciones que han hecho posibles sus contribuciones y de forma automática exportando desde Dimensions Wizard para ORCID los datos integrados y actualizados de las subvenciones de organismos financiadores de todo el mundo (Figuras 3 y 4 ).

\section{AGREGAR UNA FUENTE DE} FINANCIAMIENTO

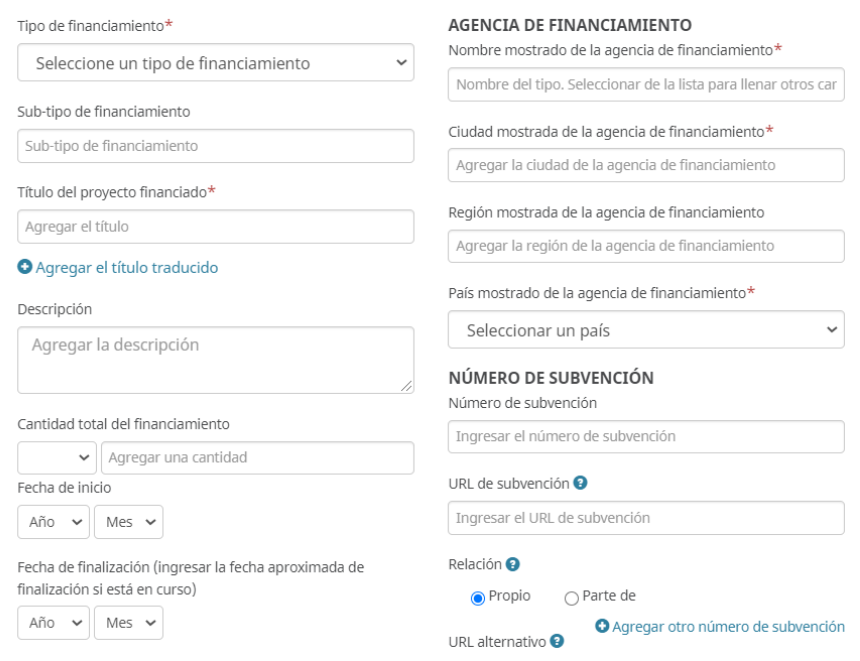

Figura 3. Registro manual de subvenciones 
DimensionsWizard for ORCID

Easily import your grants from Dimensions into ORCID.

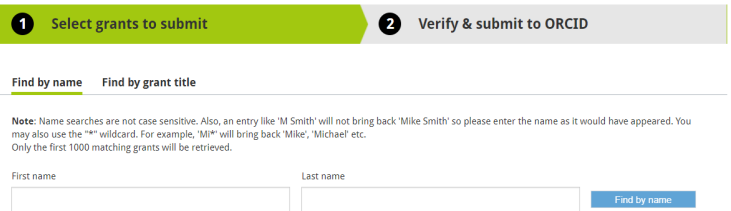

Figura 4. Registro subvención desde Dimensions Wizard

\subsection{Integración de ORCID en el sistema} de la entidad financiadora

El uso de ORCID ayuda a las entidades financiadoras a construir una infraestructura de información de investigación confiable al establecer las conexiones entre los investigadores y las subvenciones que se les conceden. Para ello es necesario que las entidades integren en su sistema de flujo de trabajo de la gestión de convocatorias la API (Interfaz de programación de aplicaciones) de ORCID para permitir el intercambio automático de información de investigación entre sistemas (Mejias, 2020). Esta integración reducirá en las solicitudes de las ayudas los errores al introducir la información de forma manual, en la concesión mejorará la accesibilidad a los datos de la subvención y en la justificación científica, la elaboración de informes de resultados más eficientes y con datos de mayor calidad (ORCID, 2021).

Con la integración de ORCID en los sistemas de las entidades financiadoras los procesos de seguimiento del flujo de la financiación se simplifican a la vez que se estandarizan. Supongamos como ejemplo un investigador que solicita a la Fundación Séneca una ayuda de uno de sus programas financiadores de I+D+i. La Fundación publica su convocatoria y en la fase de solicitud se le requiere al investigador su ID de ORCID. Para introducirlo, desde el mismo campo inicia sesión en su cuenta ORCID y autoriza al sistema de la Fundación a recopilar su identificador autenticado e intercambiar información con su registro ORCID. La Fundación recopila datos del registro del solicitante utilizando la API de ORCID y los usa para autocompletar alguno de los campos requeridos en la solicitud. Posteriormente si la ayuda es concedida la Fundación podrá incorporar los datos de la subvención al registro de ORCID del investigador, lo que le ayudará a rastrear y evaluar los resultados de la investigación financiada (ORCID, 2021).

\subsection{La membresía de ORCID para las organizaciones}

ORCID - para cubrir los gastos derivados del mantenimiento y mejora del registro, así como de los servicios que ofrece a la comunidad investigadora- depende de la financiación de la membresía de las organizaciones, entre las que se encuentran editores, entidades financiadoras, asociaciones profesionales, etc. Para los investigadores el registro y acceso a sus datos es totalmente gratuito (ORCID, 2021).

Esta organización ofrece dos niveles de membresía para las organizaciones: la básica y la premium. Ambas incluyen diferentes niveles de acceso a los datos por lo que las cuotas varían en función de la membresía contratada.

Si una organización no desea formar parte de la comunidad de miembros de ORCID puede acceder a los datos de su registro a través de una API pública que, al igual que la básica y la premium, permite que los sistemas y aplicaciones de la organización se conecten al registro ORCID e intercambien los datos del registro del investigador con su permiso previo. Además, se pueden recuperar los IDs autentificados y buscar la información que está disponible públicamente. Lo que no permite la API pública es agregar, actualizar o eliminar información del registro ORCID. Solo se podrá agregar información, y siempre contando con el permiso del usuario, con las membresías básica y premium.

\section{Otras infraestructuras de información}

ORCID y Crossref no son las únicas infraestructuras que permiten hacer un seguimiento de las subvenciones que concede una entidad para el desarrollo de la investigación. En los últimos años se ha producido un verdadero cambio en la gestión de la investigación y su difusión en plataformas ofrece nuevas oportunidades por el interés cada vez mayor de los agentes implicados para medir el rendimiento de las contribuciones de los investigadores.

En este nuevo panorama cabe mencionar la base de datos Dimensions que cubre además de de los artículos de investigación y sus citas, subvenciones concedidas, patentes, ensayos clínicos, documentos de políticas e información altmetric (Hook, Porter y Herzog, 2018). Esta base de datos ofrece tres versiones distintas, una versión gratuita (Dimensions) y dos de pago (Dimensions plus y Dimensions analytics), siendo las dos últimas las que dan acceso completo a la información de las subvenciones (entre otros documentos) y sus conexiones, además de ofrecer herramientas de análisis avanzadas, como por 
ejemplo la comparación entre organizaciones o agentes financiadores (Orduña y Delgado, 2018).

En el ámbito de la altmétrica existen los servicios de pago Plum Analytics y Altmetric Explorer de las plataformas PlumX y Almetric.com respetivamente. Ambos servicios ofrecen a la comunidad científica el acceso a información relacionada con subvenciones en investigación. Plum Analytics proporciona una gran variedad de productos que se ajustan a las necesidades de cada uno de sus clientes. En el caso de las entidades financiadoras ofrece el servicio de PlumX +Grants, el cual pone en contacto a instituciones e investigadores con una base de datos de subvenciones y proporciona evaluaciones de la actividad científica anterior, incluidos los resultados de las solicitudes concedidas (Williams, 2019).

En cuanto al servicio Altmetric Explorer dispone de tres tipos de Explorer dirigidos a varios tipos de clientes. Para las entidades financiadoras está el explorador para financiadores (Explorer for funders) cuyos datos altmétricos proporcionan un registro único de cómo se han difundido los resultados de una ayuda concedida y cuál ha sido su impacto en las redes sociales, noticias y en las políticas públicas. Asimismo, permite buscar por el beneficiario de la ayuda y áreas de conocimiento (Altmetric.com, 2021).

Para la suscripción de ambos productos hay que tener en cuenta la cobertura de estas plataformas y a quiénes van dirigidas. En el caso de PlumX sus servicios están más orientados a las organizaciones y ofrece mayor cobertura de publicaciones. En cuanto a Altmetric.com identifica, sigue y recoge métricas web a nivel de artículo y cubre más las menciones de tuits, blogs y noticias que PlumX (Ortega, 2020).

\section{Conclusiones y reflexiones finales}

El principal desafío al que se enfrentan las entidades financiadoras, como se ha comentado anteriormente, es la dificultad que tienen para seguir la trazabilidad de la financiación que conceden y medir el impacto de la misma. Partiendo del hecho de que los investigadores mencionan a sus entidades financiadoras (lo que no siempre es así a pesar de que en sus bases de convocatoria instan a sus beneficiarios a que las mencionen y cómo deben hacerlo), en los flujos de la investigación sigue habiendo lagunas que impiden el mapeo de las ayudas concedidas y de los resultados derivados de las mismas y todas apuntan a un eje principal que radica en vincular la financiación a los resultados de la investigación (De-Castro, 2018; Meadows, Brown, Demeranville y Haak, 2019). Esta conexión daría respuesta a muchos de los interrogantes que se plantean las entidades financiadoras desde que conceden las ayudas hasta que reciben su impacto porque amplía el mapeo de la financiación y sus resultados.

Como se ha visto, estas conexiones solo son posibles con los PIDs de los diferentes elementos que forman parte del ecosistema de la investigación y que actúan como puentes de intercambio de información confiable y permiten a las entidades financiadoras rastrear y analizar los datos que generan las subvenciones que conceden y sus conexiones con el resto de metadatos de la publicación. Como indica Gértrudis (2020) la principal ventaja de los PIDs para las entidades financiadoras es el tratamiento automatizado posterior de los datos que les permite hacer un seguimiento más preciso del rendimiento de la inversión realizada en las ayudas concedidas a través de los resultados de las investigaciones publicados, definir futuras políticas de I+D+i y ofrecer confiabilidad a la sociedad poniendo a su disposición dichos resultados y cumpliendo así con el principio de transparencia y rendición de cuentas.

ORCID y Crossref son infraestructuras abiertas en las que se puede registrar y compartir metadatos y PIDs entre sistemas. Aunque se centran en elementos diferentes del flujo de la investigación, ambas tienen el objetivo común de que todos los elementos participantes estén identificados y conectados entre sí de forma única y que la información confiable que arrojan estas conexiones sea peusta a disposición de la comunidad científica.

Las entidades financiadoras - tanto con la API pública que ofrece ORCID como con la consulta al Funder Registry y su ID de financiador de Crossref- pueden hacer un seguimiento de los resultados de las ayudas que han concedido de forma gratuita pero a un nivel muy básico. Si necesitan con más detalle la trazabilidad de su financiación, las entidades deben pagar una cuota en ambas infraestructuras por los servicios de registro y acceso a los datos conectados con el resto de metadatos de la publicación, lo que les permitirá elaborar informes más precisos y completos de sus subvenciones y resultados.

En el caso de Crossref la entidad financiadora puede consultar los metadatos asociados a la entidad y sus subvenciones de forma gratuita. Pero para que los datos y sus conexiones sean más fiables y específicos es necesario que sea la propia entidad la que registre las IDs de las subvenciones y sus metadatos asociados pagando para ello una cuota por cada elemento de contenido que registre en Crossref. El registro permitirá que estos datos se unan al resto de los metadatos de los miembros de Crossref lo que implica que, por 
un lado, los IDs de subvención sean incorporados por los editores en los metadatos de la publicación para que los autores puedan seleccionar los datos cuando envían sus contribuciones; y, por otro, elaborar informes de actividad más precisos con datos de mayor calidad y para el seguimiento de la financiación, información más completa sobre las vías de publicación, el impacto y los resultados de las políticas de la entidad financiadora (Crossref, 2021).

En cuanto al registro de las subvenciones en ORCID, para que una entidad financiadora pueda agregar su información tiene que ser miembro y pagar una cuota en función de la membresía contratada. En el momento en el que se concede la financiación, la entidad puede agregar la subvención al registro de ORCID del beneficiario lo que le permitirá rastrear los resultados de la ayuda concedida y automatizar los procesos de generación de informes de forma más precisa y confiable (ORCID, 2021).

Todas estas infraestructuras de información optan por un modelo de pago en la mayoría de los servicios que prestan a las entidades financiadoras, que se contradice de algún modo con su compromiso con el acceso abierto; y en el caso de PlumX y Almetric más aún, si se tiene en cuenta que gran parte de las métricas que utilizan son abiertas y las adquieren sin coste alguno (Ortega, 2020). Si partimos de la premisa de que la mayoría de las contribuciones científicas en España son el resultado de investigaciones financiadas con programas públicos regionales, nacionales y europeos, resulta incoherente que una entidad financiadora tenga que volver a pagar contratando estos servicios para acceder a los datos de sus subvenciones y resultados. Si bien es cierto que los datos que proporcionan estas infraestructuras tienen un valor añadido sobre todo por sus conexiones con otros metadatos relacionados con la publicación, no dejan de ser necesarios para que la entidad pueda hacer un seguimiento más preciso de las ayudas que concede y tomar decisiones en el diseño de futuras convocatorias imprescindibles para la generación de nuevo conocimiento científico.

Hoy en día, las agencias financiadoras están tomando conciencia de la importancia creciente de estudiar la utilización del conocimiento y los procesos de intercambio del mismo, más específicamente el impacto y el beneficio de la investigación hacia la sociedad (Comisión Europea, 2020), en el entendido de que un objetivo final de la propia evaluación es inducir procesos de cambio que modifican actitudes, creencias y acciones y consiguen influir en la mejora social vinculando los procesos de evaluación con sus resultados (Henry y Mark, 2003). Sin duda, las infraestruc- turas de información como ORCID y Funder Registry de Crossref están orientadas a la consecución de dicho propósito y permitirán a las entidades financiadoras, además de definir mejor la trazabilidad de las ayudas concedidas y de sus resultados científicos, identificar ideas y resultados nuevos, respuestas a retos sociales, económicos y culturales, detectar la generación de valor a través del conocimiento científico, el beneficio social de las inversiones públicas en I+D, y la explotación del potencial de innovación comercial y social de las actividades que financian. En definitiva, dichas infraestructuras ofrecerán a las entidades la posibilidad de adoptar decisiones mejor informadas para la consecución de sus fines.

Dejaremos para próximas investigaciones el análisis de las oportunidades que ofrecen para las entidades financiadoras las bases de datos de Dimensions plus y Dimensions analytics y los productos Plum Analytics y Altmetric Explorer de los agregadores de métricas PlumX y Almetric.com respetivamente.

\section{Referencias}

Altmetric.com (2021). Researcher Data Access Program. https://www.altmetric.com/audience/funders/ (01.04.2021)

Arnold, Carrie; Dahan, Maytal; Hart, David L. y Soriano, Ester (2019). ORCID Integration into Science Gateways. // Proceedings of the Practice and Experience in Advanced Research Computing on Rise of the Machines (learning) (PEARC '19). Association for Computing Machinery, New York, NY, USA. 47, 1-4. https://dl.acm.org/doi/10. 1145/3332186.3332227. (23.03.2021).

Bilder, Geoffrey y Hendricks, Ginny (2017). Global Persistent Identifiers for grants, awards, and facilities. // Blog Crossref. https://www.crossref.org/blog/global-persistent-identifiers-for-grants-awards-and-facilities/ (25.03.2021).

Comisión Europea, (2020). Horizon 2020 Swafs Evaluation: H2020-19-2018-2019 - Tacking stock and re-examining the role of Science Communication (RIA). https://ec.europa.eu/info/sites/info/files/h2020-swafs-19-2018-2019__ria_-_topic_briefing_-_raluca_iagher.pdf. (29/05/2021).

Crossref (2019). Annual membership fees (funders). https://www.crossref.org/fees/\#annual-membership-feesfunders (30.03.2021).

Crossref (2021). Funder advisory group. https://www.crossref.org/working-groups/funders/ (29.03.2021).

Crossref (n.d.). Funder registry. https://www.crossref.org/p dfs/about-funder-registry-spanish.pdf (29.03.2021).

Crossref (2021). Registering research grants. https://www. crossref.org/community/grants/ (30.03.2021).

De-Castro, Pablo (2018). Importancia de la información de financiación. // Anuario ThinkEPI. ISSN 2564-8837. 12, 258-264 (April 2018). https://doi.org/10.3145/ thinkepi.2018.39 (25.02.2021).

De-Castro, Pablo (2017). The quest for funding information. euroCRIS blog. // Current research information systems, 18 agosto. http://eurocris.org/blog/quest-funding-information (04.03.2021).

Gértrudix Barrio, Manuel (2020). El uso de los identificadores persistentes (PIDs) de instituciones en los artículos científicos facilita el seguimiento y trazabilidad de las 
ayudas. Blog de Ciberimaginario. https://tinyurl.com/ y5s1973v (17.03.2021).

Henry, Gary-T.; Mark, Melvin-M. (2003). Beyond Use: Understanding Evaluation's Influence on Attitudes and Actions. // American Journal of Evaluation. ISSN 1098-2140. 24:3, 293-314. https://doi.org/10.1177/109821400302400302 (29/05/2021)

Hook, Daniel W.; Porter, Simon J. y Herzog, Christian (2018). Dimensions: Building Context for Search and Evaluation. // Front. Res. Metr. ISSN 2504-0537. 3:23 (August 2018). https://www.frontiersin.org/articles/10.3389/frma.2018.0 0023/full (24.03.2021).

Lammey, Rachael (2020). Solutions for identification problems: a look at the Research Organization Registry. // Sci Ed. ISSN 2288-7474. 7:1 (January 2020) 65-69. https://doi.org/10.6087/kcse.192 (24.03.2021).

Meadows, Alice; Brown, Josh; Demeranville, Tom y Haak, Laurel L. (2019). ORBIT Funder Reporting Survey Report. ORCID. https://doi.org/10.23640/07243.9149240.v1 (28. 03.2021).

Meadows, Alice; Haak, Laurel L. y Brown, Josh (2019). Persistent identifiers: the building blocks of the research information infrastructure. // Insights. ISSN 2542-6648. 32:1 9. http://doi.org/10.1629/uksg.457 (30.03.2021).

Meadows, Alice y Haak, Laure L. (2018). How persistent identifiers can save scientists time. // FEMS Microbiology Letters. ISSN 0378-1097. 365:15 (August 2018) https:// doi.org/10.1093/femsle/fny143 (24.03.2021).

Mejias, Gabriela (2020). Improving Funding and Publishing Workflows with PIDs. // Information Services \& Use. ISSN 1875-8789. 40:4 307-311.https://doi.org/10.3233/ISU200074 (25.03.2021).
ORCID, (2021). Funder and Grants. https://info.orcid.org/documentation/workflows/funding-workflow/ (27.03.2021).

ORCID, (2021). ORCID for Funding Organizations. https://info.orcid.org/orcid-for-funding-organizations/ (28. 03.2021).

ORCID, (2021). ORCID Membership. https://info.orcid.org/about-membership/ (27.03.2021).

Orduña-Malea, Enrique; Delgado-López-Cózar, Emilio (2018). ¡Viva la competencia! Nuevas dimensiones para la búsqueda y evaluación de la información científica. /l Anuario ThinkEPI. ISSN 2564-8837. 12 (Abril 2018) 304310. https://doi.org/10.3145/thinkepi.2018.45. (23.03.2021).

Ortega, J. L. (2020). Altmetrics data providers: a meta-analysis review on the coverage of metrics and publications. // EI Profesional de la Información. ISSN 1699-2407. 29:1. http://profesionaldelainformacion.com/contenidos/2020/ ene/ortega.pdf (01.04.2021).

Kiley, Robert; Frentrop, Nina y Hendricks, Ginny (2018). Wellcome explains the benefits of developing an open and global grant identifier. Blog Crossref. https://www.crossref.org/blog/wellcome-explains-the-benefits-of-developing-an-open-and-global-grant-identifier/ (24.03.2021).

Williams, Ann E. (2019). Exploring the utility of an emerging altmetric platform: a SWOT analysis of Plum Analytics. // Digital Library Perspectives. ISSN 2059-5816. 35:3/4 (November 2019) 193-204. https://doi.org/10.1108/DLP-082019-0031 (23.03.2021).

Enviado: 2021-04-08. Segunda versión: 2021-05-31. Aceptado: 2021-06-01. 